\title{
Étude expérimentale des rétrocourants sous-foliaires dans les Bryophytes immergées : implications écologiques
}

\author{
P. Devantéry ${ }^{1}$
}

Mots clés : microcourants, effets tourbillonnaires, rétrocourants, couche-limite, bryophytes.

L'observation de dépôts sédimentaires et de faune sous les feuilles de Bryophytes immergées dans les rivières suggérait l'existence d'une modification du courant au sein des touffes de mousse. Des expériences ont été réalisées, en rivière artificielle, sur Platyhypnidium riparioides. Un filet de colorant, introduit dans le dispositif expérimental, a servi de traceur pour visualiser les phénomènes hydrauliques. La présence d'un brin de mousse, muni d'une seule feuille entraine, à l'arrière de cette feuille, la formation de deux tourbillons symétriques. Entre les deux se crée un rétrocourant orienté vers la feuille qui se ralentit progressivement et s'incurve vers sa zone d'insertion. La genèse hydrodynamique de ce rétrocourant a été étudiée en fonction de l'importance de la touffe de mousse et de la vitesse du courant. Ce phénomène a été également observé sur deux autres espèces de Bryophytes de morphologie foliaire différente. Quelques implications écologiques de ces rétrocourants sont envisagées.

\section{Experimental study of sub-foliar retrocurrents in underwater Bryophytes : ecological implications}

Keywords: microcurrents, twirling effects, retrocurrents, boundary layer, bryophytes.

The observation of sedimentary deposits and fauna below the leaves of underwater bryophytes in streams suggested a modification of the internal current inside the mosses. Experimental observations were realized in an artificial stream with Platyhypnidium riparioides. A coloured liquid was used as a tracer to reveal the hydraulic phenomena. A blade of moss with a single leaf revealed a symmetrical twirling formation behind it. Between them is established a retrocurrent, in the direction of the leaf, which progressively slowed down, turning towards foliar insertion. The hydrodynamic genesis of this retrocurrent has been studied in two different bryophyte species that differ in the morphology of their leaves. Some ecological implications of these retrocurrents are envisaged.

\section{Introduction}

La présente étude expérimentale succède à un travail antérieur de terrain sur «l'action des courants sur la faune d'une mousse immergée» (Devantéry 1987). A l'origine, nos mesures de terrain - bien qu'échelonnées axialement de 5 en $5 \mathrm{~mm}$, dans des prélèvements de $5 \mathrm{~cm}^{2}$ - confrontées aux inventaires de faune, nous apparaissaient insuffisamment explicatives. Le manque d'homogénéité des courants, face à une présence d'organismes de l'ordre du millimètre semblait en cause.

1. Hermitage B.P. 9 - F - 42405 Saint-Chamond.
De plus, l'origine d'éléments faunistiques particuliers, précisés plus loin ( $\$ 4)$; ainsi que la présence sous-foliaire de détritus souvent surabondants demeuraient énigmatiques.

Des questions similaires $(\S 4)$ émanent de maintes recherches antérieures concernant le rôle du courant dans les mousses. Citons les études de Johnson (1978) sur le rapport entre vitesses et dimensions des particules et celles de Scott (1958) et Décamps et al. (1972) montrant la plus grande résistance au courant chez des larves vivant sur des surfaces recouvertes de mousses. Angelier et al. (1985) confirment ce fait à propos des Hydracariens.

La morphologie propre de la mousse immergée et la forme avec convexité de type nacelle de chacune des feuilles jouent un rôle face au courant, qu'il importe de 
préciser expérimentalement. Dans ce domaine expérimental, plusieurs auteurs ont apporté leur contribution, notamment Dorier \& Vaillant (1954), Bournaud (1963), Trivellato \& Décamps (1971) et Illies et al.(1974).

L'originalité de notre travail portant sur Platyhypnidium riparioides.(Hedwig 1801) tient précisément au fait d'avoir privilégié l'observation d'une feuille isolée, sur sa tige. Il s'agissait donc, de contrôler visuellement les modifications subies par un courant suffisamment homogène rencontrant une feuille de mousse en position naturelle. Une comparaison a été établie pour d'autres espèces de mousses (Fontinalis antipyretica). Les données obtenues ont conduit vers une vérification des lois observées pour une seule feuille à l'ensemble du brin de mousse et à la strate moussue.

\section{Appareillage et méthode (Devantéry 1987)}

Les expériences ont été réalisées en micro-rivière artificielle, sous tube de verre de section carrée, ramenée à $1 \mathrm{~cm}^{2}$, réduction facilitant l'accès aux courants laminaires (Ambühl 1959). Ce tube d'observation (Fig. 1) est précédé et suivi d'éléments visant à l'arrivée d'un courant suffisamment corrigé et modulable. Pour éviter la formation de bulles d'air, l'eau de renouvellement est maintenue à température de laboratoire en réservoir annexe. La mise en évidence des courants observés est rendue possible par l'intervention d'un mince filet de colorant à débit réglable, continu ou ponctuel et à direction d'arrivée adaptable (Fig. 1).

Maintenue par un support, la fraction de tige dépouillée de ses feuilles, sauf de la plus antérieure, est glissée axialement jusqu'à mi-course dans le micro-tube d'observation. Des échantillons de tiges, puis de brins conservant toutes leurs feuilles sont également utilisés. L'échantillon en place est observé au binoculaire, soit en vue de dessus, soit en vue latérale, à l'aide de miroirs et éclairages appropriés. Le déroulement des observations est enregistré par photos, films et vidéogrammes, ce qui facilite l'analyse. Le filmage (en format réduit) a permis, par superposition de plans consécutifs, des reconstitutions de mouvements.

Des vitesses de courant trop élevées ont montré une continuité dans les courants secondaires obtenus. Les observations de base ont donc porté surtout sur-des vitesses lentes, de 6 à $10 \mathrm{~cm} / \mathrm{s}$. Celles-ci respectent da-

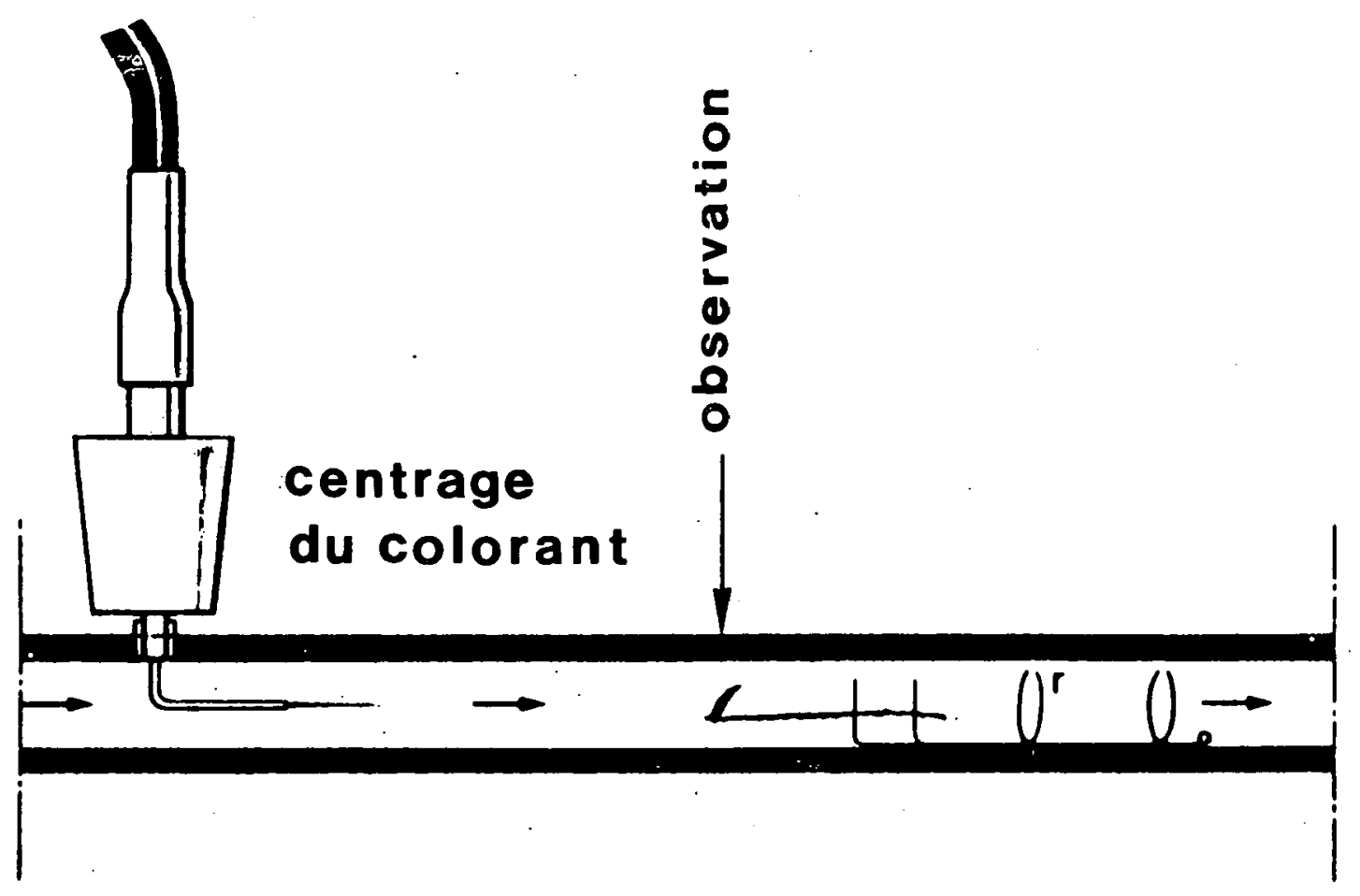

Fig. 1. Tube (microrivière artificielle) d'observation - avec échantillon en place et distributeur de colorant.

Fig. 1. Observation pipe (artificial microriver) with sample on the spot and distributor of the dye. 
vantage le milieu laminaire et sont plus finement révélatrices de la genèse de ces courants. Des contrôles en vitesses rapides ont été cependant opérés ( $\$ 3.4$.). La mesure précise des vitesses est effectuée en direct, au chronomètre, entre deux points de la micro-rivière, l'un étant le point de sortie d'une mini-gouttelette de colorant, l'autre l'extrême limite aval du tube d'observation.

Pour un contrôle des effets de freinages $(\S 3.6$.) nous avons monté un canal découvert en tôle mince d'aluminium (largeur $2 \mathrm{~cm}$, hauteur $3 \mathrm{~cm}$, longueur $30 \mathrm{~cm}$ ) avec mini-pince réglable, pour la fixation d'un bouquet de mousses. Ce canal présente une entrée large d'eau du robinet et une évacuation suffisante.

\section{Résultats}

D'apparence tout d'abord complexe, les effets de turbulence subits par le courant contre la feuille, se prêtèrent progressivement à une certaine généralisation.

\subsection{Mouvements tourbillonnaires primaires}

La feuille de Platyhypnidium riparioides (Fig. 2) est en forme de nacelle, plus ou moins divergente en direction de l'apex. Dans un courant modéré $(6 \mathrm{~cm} / \mathrm{s})$ venant enserrer ses flancs côté convexe, le flux le plus externe continue son chemin en ligne droite, alors qu'une tendance progressive à l'enroulement se manifeste en bordure du limbe, en fonction du frottement et du freinage subits par chaque couche liquide. Il se crée ainsi un mouvement tourbillonnaire (Fig. 3).

On observe en effet la formation bi-latérale et symétrique de tourbillons de sens antagonistes (Fig. 3. 2). Ces tourbillons tendent à s'allonger selon la direction du flux général, en fonction de l'inclinaison de la feuille et de la vitesse du courant. Ils s'élargissent et se creusent, en fonction de cette vitesse. La feuille schématisée figure 3-1 est peu inclinée et cet allongement difficilement observable. Par contre, une coloration par un mince filet ( $\mathrm{z}$ ) côté tige des seuls tourbillons, ré-

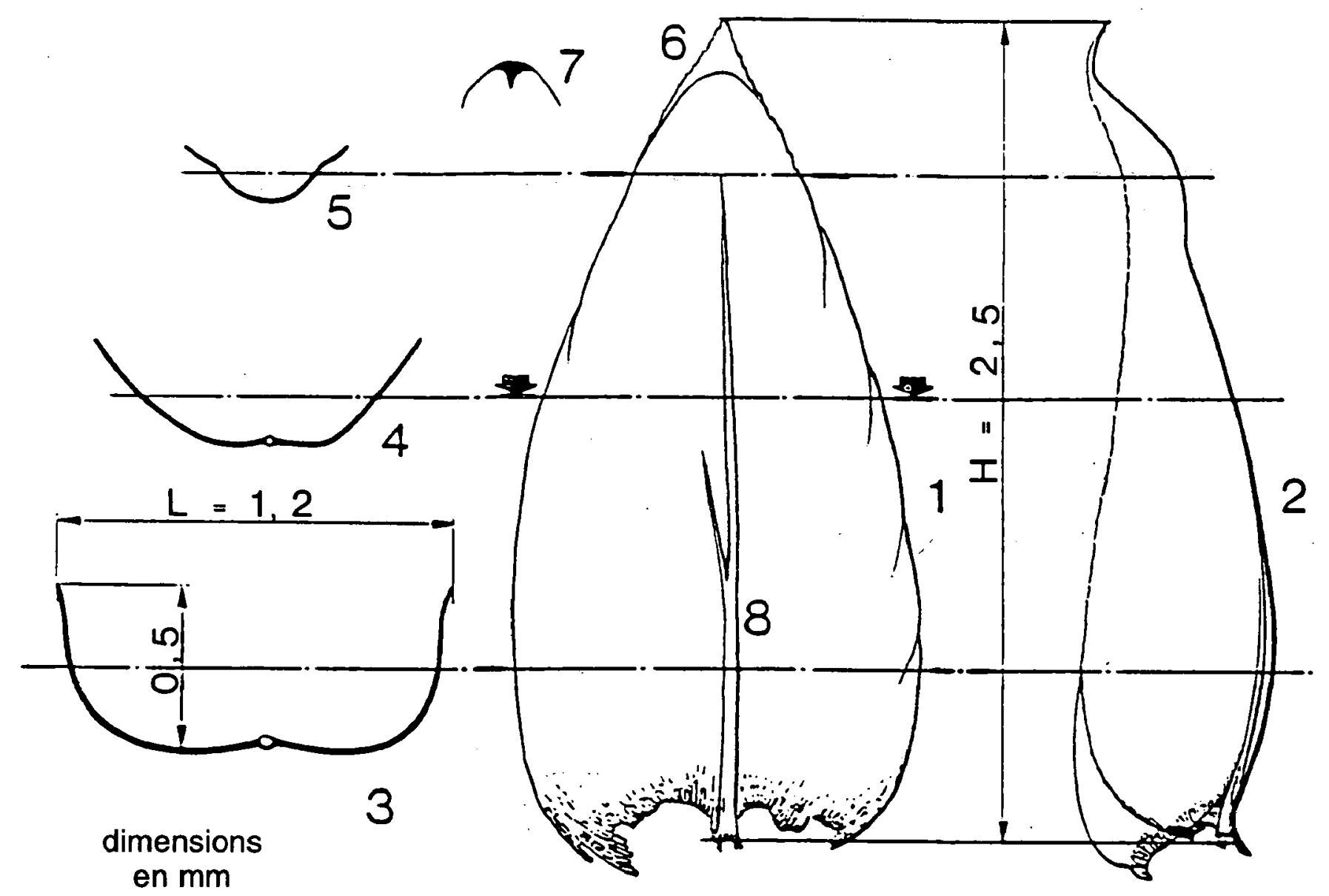

Fig. 2. Vues de face, de profil et en coupes d'une feuille moyenne de Platyhypnidium riparioides.

Fig. 2. Front, profile and cross-section views of a middle-sized leaf of Platyhypnidium riparioides. 

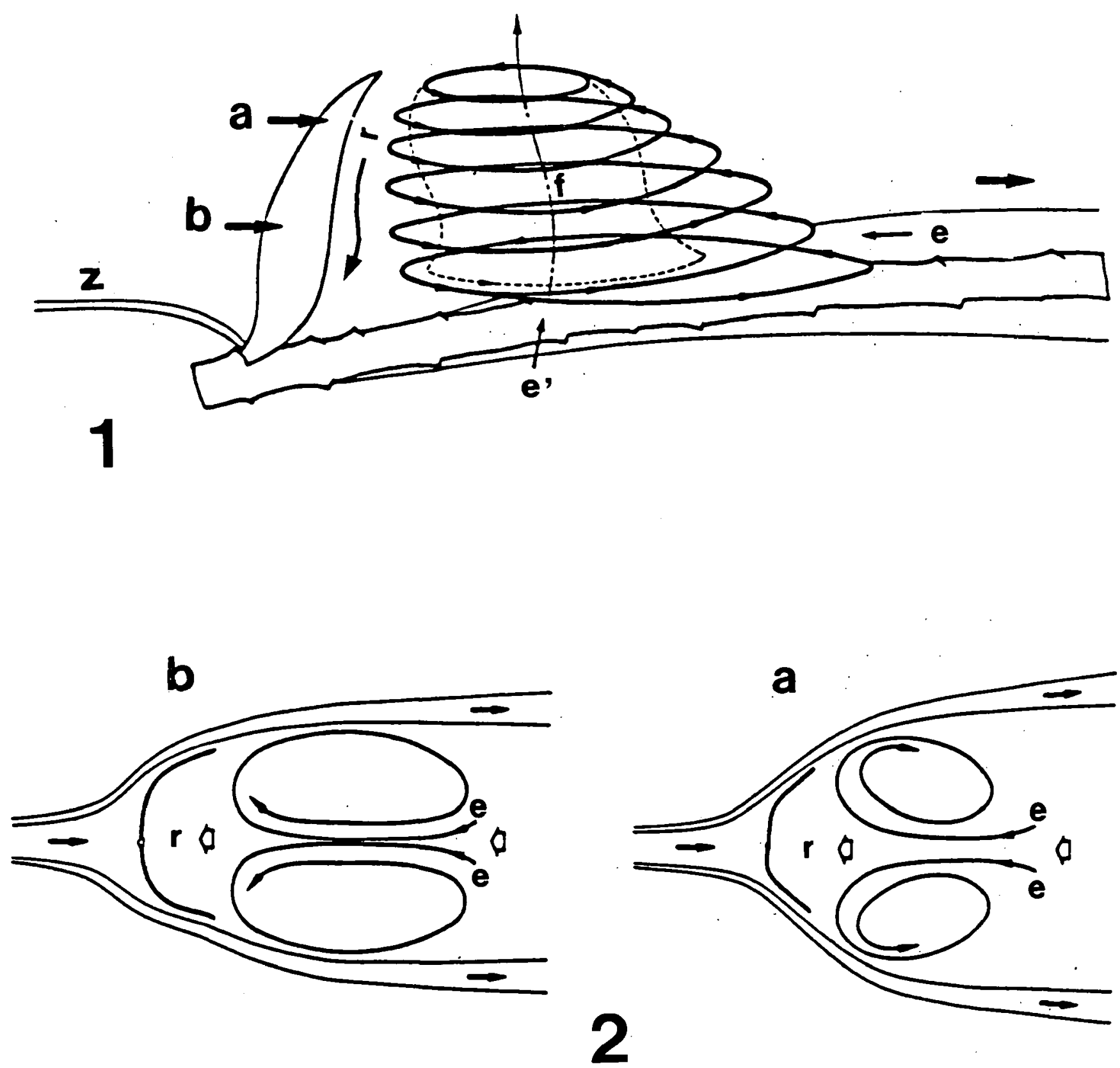

Fig. 3. Caractéristiques des (2), tourbillons engendrés par le courant coiffant la feuille de Platyhypnidium riparioides, placée dans le tube d'observation (Fig. 1).

1 : vue de côté, sens de circulation.

2 : sections selon les plans $a$ et $b$ de (1) des deux tourbillons antagonistes

$z, e, e^{\prime}$ : entrée des colorants

$r$ : courant récurrent (ou rétrocourant)

f : sens de cheminement du colorant

Fig. 3. Characteristics of the whirhpools caused by the current covering the observed leaf of Ptaryhypnidium riparioides.

1: direction of the circulation from the side

$2:$ theoretical sections according to the driving plans $a$ and $b$ of $(1)$ the two antagonist whirlpools

$z, e, e^{\prime}:$ entry of the dye

$\mathbf{r}$ : recurrent driving (or retrocurrent)

$f$ : direction of the following dye 
vèle aisément le départ du tourbillon ( $\left.e^{\prime}\right)$ et son cheminement (f) extrêmement ralenti (en perte de coloration) en direction de l'apex, dès suppression du filet colorant.

\subsection{Rétrocourant}

Ces mouvements tourbillonnaires primaires sont le moteur d'un autre courant, facilement observable, à valeur écologique évidente. Son sens est opposé au flux général (r, Fig. 3) et il progresse sous la feuille en se ralentissant de l'apex vers l'insertion de celle-ci.

Une schématisation par superposition de plans filmés successifs a permis d'entrevoir la genèse de ce rétrocourant. Les deux tourbillons antagonistes agissent par effet de laminoir, élevant et ramenant le fluide des rouleaux R et R' (Fig. 4) vers la cavité foliaire et en direction de l'insertion sur la tige. Le guidage de ce «rétrocourant» dépend de la morphologie et de l'inclinaison même de cette cavité. Une partie du ralentissement observé paraît due à la concavité très progressive de la feuille réceptrice et il est assimilable à «un effet ralentisseur de canal de largeur croissante» (Ambühl 1959).

Le ralentissement principal est dû au freinage tourbillonnaire du mouvement propre, en direction de l'apex. En observations latérales, nous avons vérifié la progression excessivement lente du fluide en direction de l'apex, dans chacun des deux tourbillons (Fig. 3. 1). Rappelons qu'une telle lenteur est due au fait que «les mouvements tourbillonnaires s'accompagnent de pertes d'énergie puisqu'ils s'accompagnent de glissements relatifs des filets qui font intervenir des forces de viscosité» (Larras 1974).

C'est ici qu'interviennent l'inclinaison et la morphologie sous-foliaire, transformant un courant très lent en rétrocourant dégressif durant cette remontée tournante, inversant la direction de la partie du flux happée et ainsi déviée par cette cavité limbale (Fig. 4). La variabilité individuelle de cette morphologie foliaire induit ainsi des variantes dans la modulation de ces courants. Le rétrocourant serait d'ailleurs presque nul dans le cas extrême de feuilles filiformes.

\subsection{Généralisation du phénomène}

Le principe étant connu, il est aisé de constater, expérimentalement, la constance de ces mêmes effets tourbillonnaires sur un brin feuillu. Selon la distribution du filet colorant, chaque feuille révèle à son tour, un courant de pénétration sous-jacent de même type, en sens inverse du courant général, compte tenu de certaines interférences ou conjugaisons d'effets chez des feuilles trop voisines. Le ralentissement final de chaque cheminement paraît d'autant plus décelable que la feuille est plus inclinée. La figure 5, en superposition de plans consécutifs, fournit un exemple assez typique, par coloration locale de ces courants, en fait omniprésents et continus, en fonction de l'exposition effective de chaque feuille au courant. Seule une représentation cinématique peut en rendre compte aisément.

A titre d'exemple, avec un écoulement, longeant les faces convexes des feuilles, de $14 \mathrm{~cm} / \mathrm{s}$ (Fig. 6) une vitesse de rétrocourant moyenne de $0,9 \mathrm{~cm} / \mathrm{s}$ a été relevée sur $2 \mathrm{~mm}$ (a... e Fig 6.2) d'entrée sous-foliaire repérée par plans films consécutifs au $1 / 18 \mathrm{sec}$. Avec une progression calculée vue par vue, (raison repérée empirique de $1 / 1,2$ ) on passerait déjà à des vitesses de $1,2 \mathrm{~cm} / \mathrm{s}(\mathrm{ab}), 1 \mathrm{~cm} / \mathrm{s}$ (bc), $0,8 \mathrm{~cm} / \mathrm{s}$ (cd), $0,65 \mathrm{~cm} / \mathrm{s}$ (de),... et ainsi de suite. Ainsi, à l'approche des fins reliefs du fond de la feuille, la couche-limite est plus rapidement effective. C'est l'inverse en face convexe. La persistance du colorant dans les seuls rétrocourants situe d'ailleurs l'ordre de grandeur de ce ralentissement.

Il faut tenir compte du fait que le rétrocourant naît de la pression des remontées inter-tourbillonnaires dans le fond de la feuille (Fig. 4). De semblables décompositions de mouvement réalisées au magnétoscope montrent le très grand ralentissement final mais aussi que le degré d'inclinaison de la feuille vers la tige favorise une déviation vers la cavité formée par l'insertion foliaire qui devient ainsi encore plus créatrice d'inertie, comme en témoigne une plus facile stagnation de colorant. Les repères utilisés (Fig. 6) n'expriment donc pas ici la «pointe» du ralentissement. C'est avec des feuilles de moins en moins inclinées que de telles approches de la couche-limite tendent au contraire à s'étaler en direction de l'apex, un éventail de localisation des ralentissements devenant ainsi envisageable.

\subsection{Influence de la vitesse du courant principal}

Une accélération (d'abord modérée) du courant ne semble pas supprimer le rétrocourant, décelable en dépit de l'augmentation de la turbulence et dès lors d'une plus grande mobilité et dispersion des filets de colorant. La vitesse de ces rétrocourants semble augmenter avec la vitesse du courant principal. Elle devient toutefois de plus en plus difficile à évaluer quand ce courant principal s'accélère (§ 3.6.). Le rôle de l'inclinaison et de la morphologie des feuilles ne semble pas négligeable. 


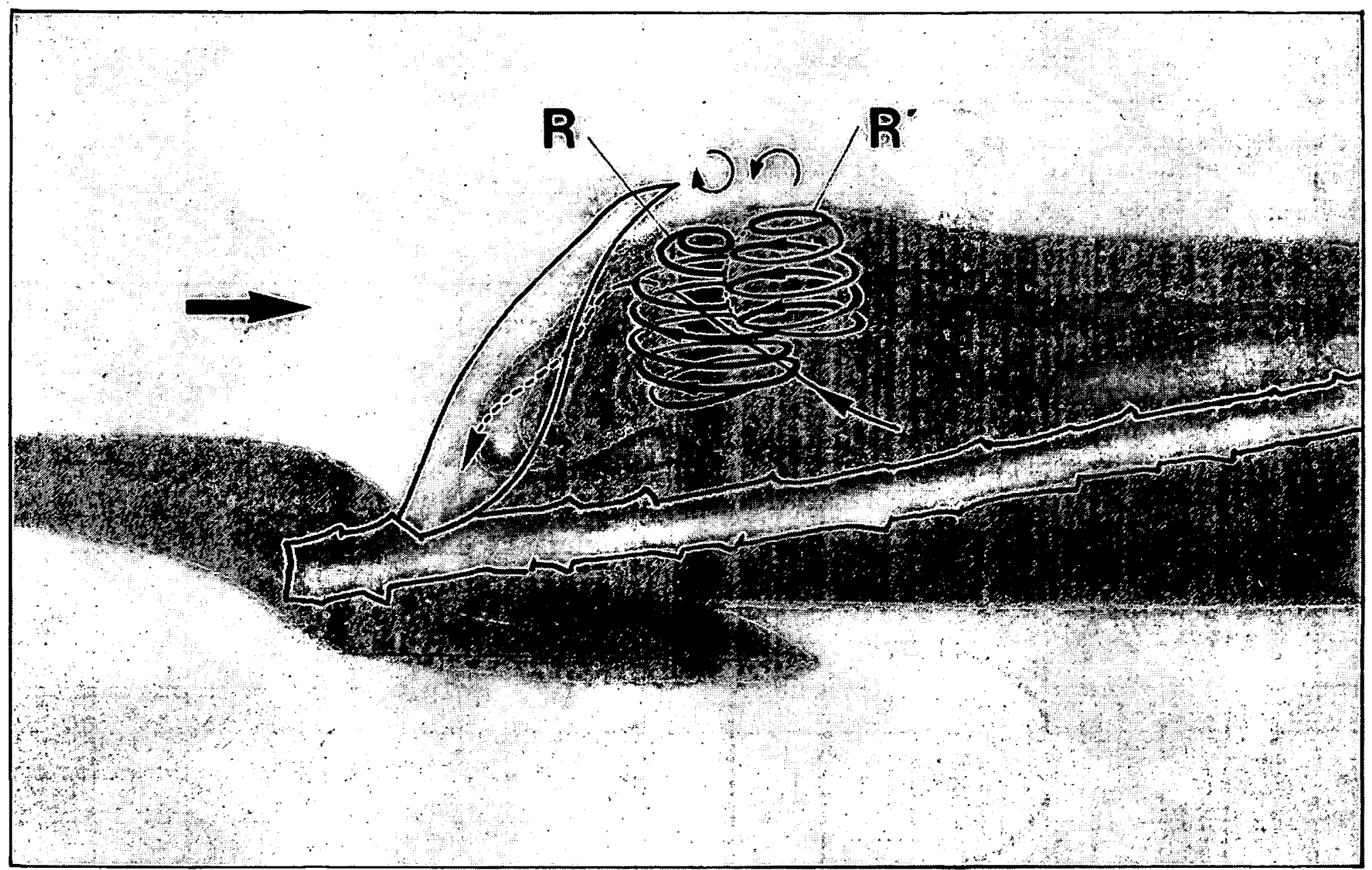

Fig. 4. Synthèse en superposition de plans-film, de l'amorce ponctuelle d'un flux récurrent. Remontée des vagues colorées (selon des instantanés successifs au $1 / 18 \mathrm{~s}$ ) déviées vers le fond de la feuille (flèche). Successivement, fin d'un des cycles et pénétration du colorant-témoin suivant, mais en commande manuelle, non homocinétique.

$R$ : tourbillon de gauche

$R^{\prime}$ : tourbillon de droite (vue en direction du courant principal)

Flèche en gras : flux général, ou principal ; flèche en maigre : entrée du colorant

Flèche circulaire : sens giratoire des tourbillons

Flèche pointillée : sens du rétrocourant

En arrivées ponctuelles, les vagues de colorant englobent et entraînent la masse de colorant concrétisant l'un et/ou l'autre tourbillon(s) qui ainsi disparai(ssen)t optiquement.

Un phénomène dû à la viscosité plus élevée du colorant, laquelle, tout en permettant l'examen localisé de la cause (tourbillons) et de l'effet (rétrocourants) gêne la simultanéité de l'observation. L'esquisse des tourbillons tend ici à y remédier.

Fig. 4. Synthesis superposing film-plans on the punctual start of a recurrent ebb. Ascent of the coloured waves (according to successive snapshots $1 / 18 \mathrm{~s}$ ) shifted towards the bottom of the leaf (arrow).Successively, end of the cycles and penetration of the following experimental dye, but manually led, not homokinetically.

$\mathrm{R}$ : left twirling

$\mathrm{R}^{\prime}$ : right twirling (seen in the direction of the principal current)

Thick arrow : general ebb or principal ; thin arrow : entry of the dye

Circular arrow : gyratory sense of the twirlings

Dotted arrow : sense of the retrocurrent

With punctual arrivals, the waves of the dye incorporate and drag along the mass of the dye materializing one or both twirling, which, in such a way optically disappear.

A phenomenon caused by the higher viscosity of the dye, which, through allowing the localized survey of the cause (twirlings) and of the effect (retrocurrents):hinders the simultaneousness of the observation. The sketch of the twirlings tends here to find a remedy for it. 


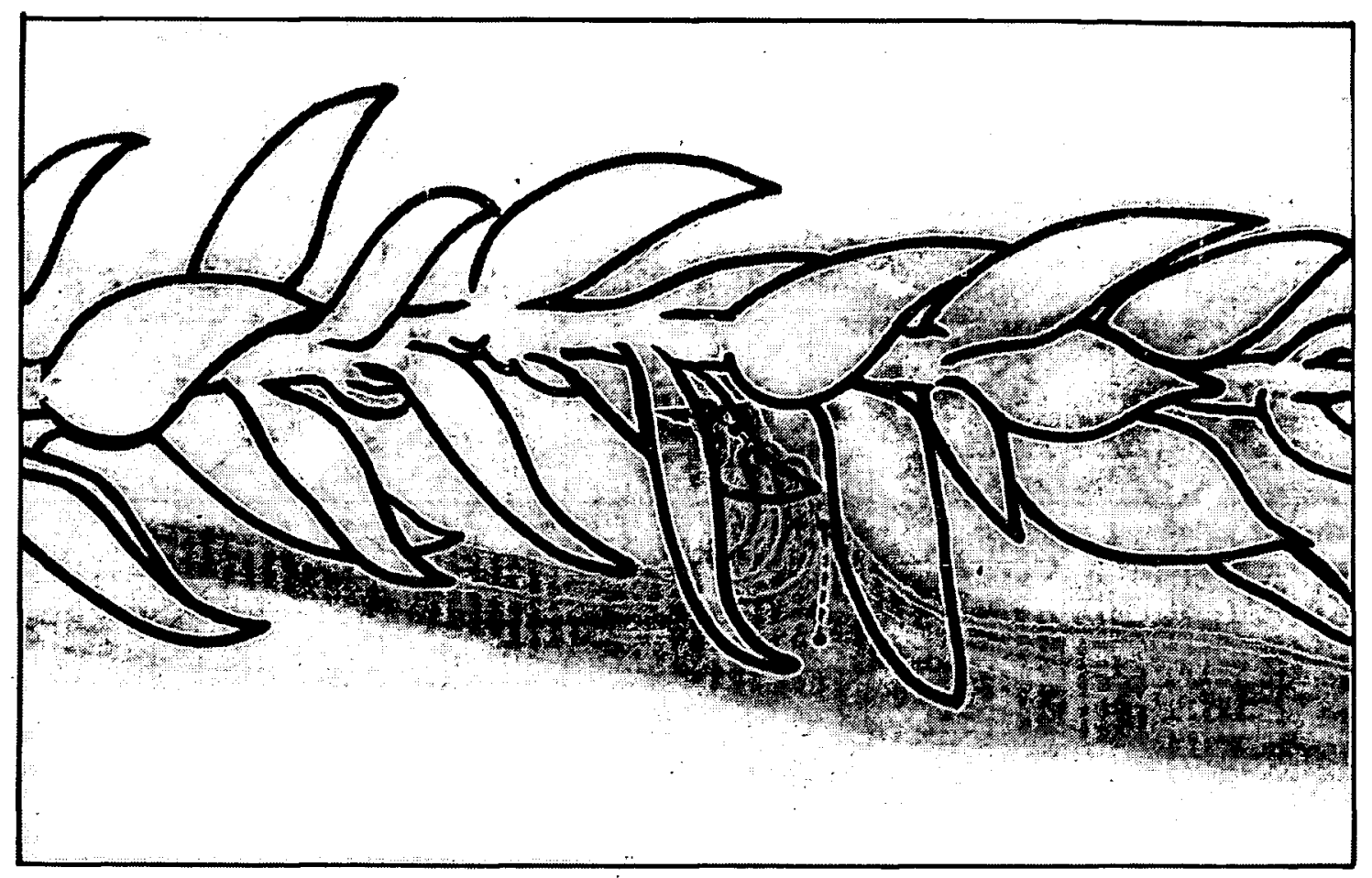

Fig. 5. Exemples de contrôle, par filet de colorant balladeur, de la formation effective, sous chacune des feuilles successives, des rétrocourants.

Fig. 5. Examples of controlling by trickle of wandering dye of the effective formation, under each of the successive leaves of the retrocurrents.

\subsection{Influence des rétrocourants sur le courant principal}

L'influence en retour des rétrocourants sur le flux principal - ou plus indirectement celle des tourbillons qui les génèrent - est loin d'être négligeable. L'analyse par repérages sur quatre vues filmées consécutives süperposées, du déplacement vers une feuille montée d'une goutte de colorant.en fournit une première idée (Fig. 7). La déformation est presque nulle avant la rencontre de la feuille; puis l'avance est régulière du côté sans feuille alors que le freinage est considérable du côté opposé, affrontant ou frôlant la zone tourbillonnaire foliaire. Avec un brin conservant toutes ses feuilles, à plus forte raison en pleine strate, le potentiel de ces freinages omniprésents doit être très important. Sur le terrain, ces ralentissements devraient normalement se multiplier, pour une parcelle liquide donnée, en déplacement, au prorata de ses rencontres plus ou moins directes et nombreuses avec la convexité de ces feuilles de mousse. Il reste cependant le problème de l'hétérogénéité du milieu ( $\$ 3 \%$. $)$.

\subsection{Référence aux mesures de terrain}

Les mesures de terrain ne peuvent évaluer directement ces rétrocourants ; mais on peut se demander toutefois ce que reflètent les mesures intercalaires, prises par exemple, par un tube Darcy assez réduit (Devantéry 1987). La proximité des freinages foliaires étant en cause, les dimensions des espacements intercalaires entre les brins dans la strate sont donc facteurs d'hétérogénéité dans la représentativité des vitesses mesurées. Il serait habituellement difficile, pour un prélèvement donné de mousse, d'apprécier la distribution de ces espaces et d'en tenir compte.

En fonction de ces critères, les vitesses de l'eau mesurées en strates plus denses refléteront davantage les ralentissements sous-foliaires que celles mesurées en 


\section{repères de vitesse ambiante}
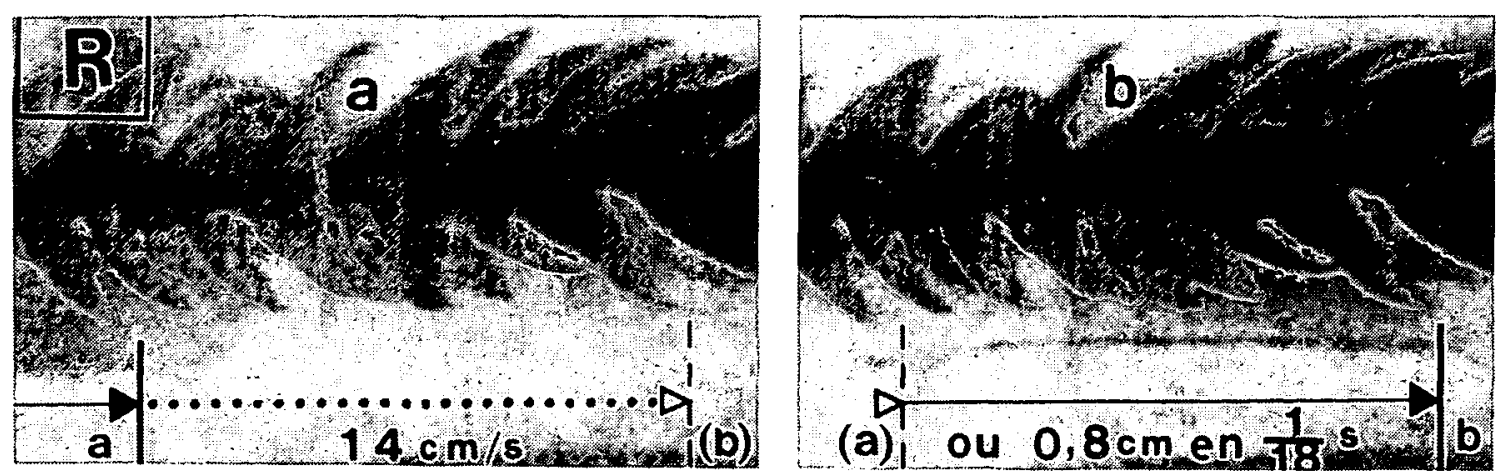

\section{repères de vitesse récurrente [colorantsicinonreproduits]}
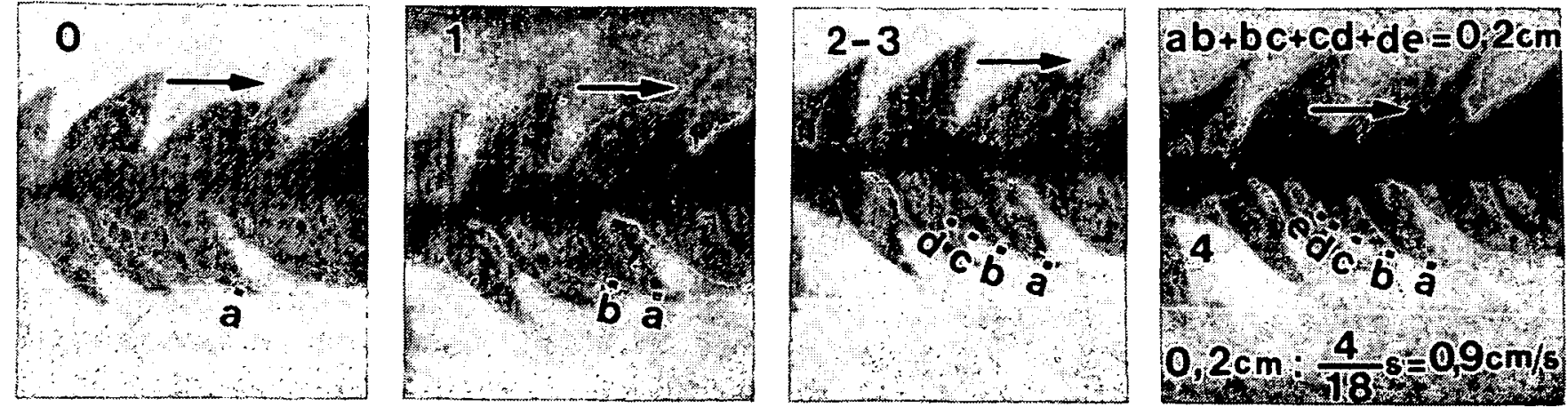

Fig. 6. Essais de comparaison (1.) entre vitesse ambiante et (2.) vitesse récurrente, selon repères portés sur plans filmés consécutifs.

1. a : front de départ du filet colorant-témoin (visible)

b : front d'arrivée de ce filet, au $1 / 18 \mathrm{~s}$.

2. flèche : direction du courant général

ab, bc, cd, de, déplacements frontaux successifs du colorant-repère (invisible ici) à chaque succession de plans filmés, au $1 / 18 \mathrm{sec}$.

Fig. 6. Tests in comparing (1) ambient speed and (2) recurrent speed according to marker written on consecutive filmed plans.

1. a : front of departure of the (visible) experimental dye

$\mathrm{b}:$ front of arrival of this dye, in $1 / 18 \mathrm{~s}$.

2. arrow : sense of the general current

$a b, b c, c d$, de, successive frontal shifting of the dye (here invisible) at every succession of filmed plans, in $1 / 18 \mathrm{~s}$.

mousses grêles, aux brins plus distants, souvent très fréquentes en courants rapides.

En canal découvert ( $\$ 2$.) nous avons comparé les vitesses d'entrée et de sortie d'un courant traversant un bouquet de brins de Fontinalis antipyretica. Selon la densité de cette mousse, des différences de vitesses entrée-sortie apparaissaient évidemment, mais avec des écarts en sortie souvent considérables suivant le point de contrôle. L'hétérogénéité se manifeste surtout entre les filets intercalaires, qui sont cependant plus linéaires. De ce fait, le brassage paraît restreint; donc, les zones protégées grâce à la forte densité de feuilles seront plus nombreuses; ces zones sont susceptibles d'être très recherchées par les organismes.

\section{Comparaison avec d'autres espèces de mousses}

Notre expérimentation s'est volontairement limitée à deux autres espèces présentes dans la rivière explorée, le Gier : Fontinalis squamosa et Fontinalis antipyretica. F. squamosa (Fig. 8. 2), malgré sa forme plus fuselée à section plus circulaire, rappelle davantage la morphologie de notre modèle $P$. riparioides. Malgré 


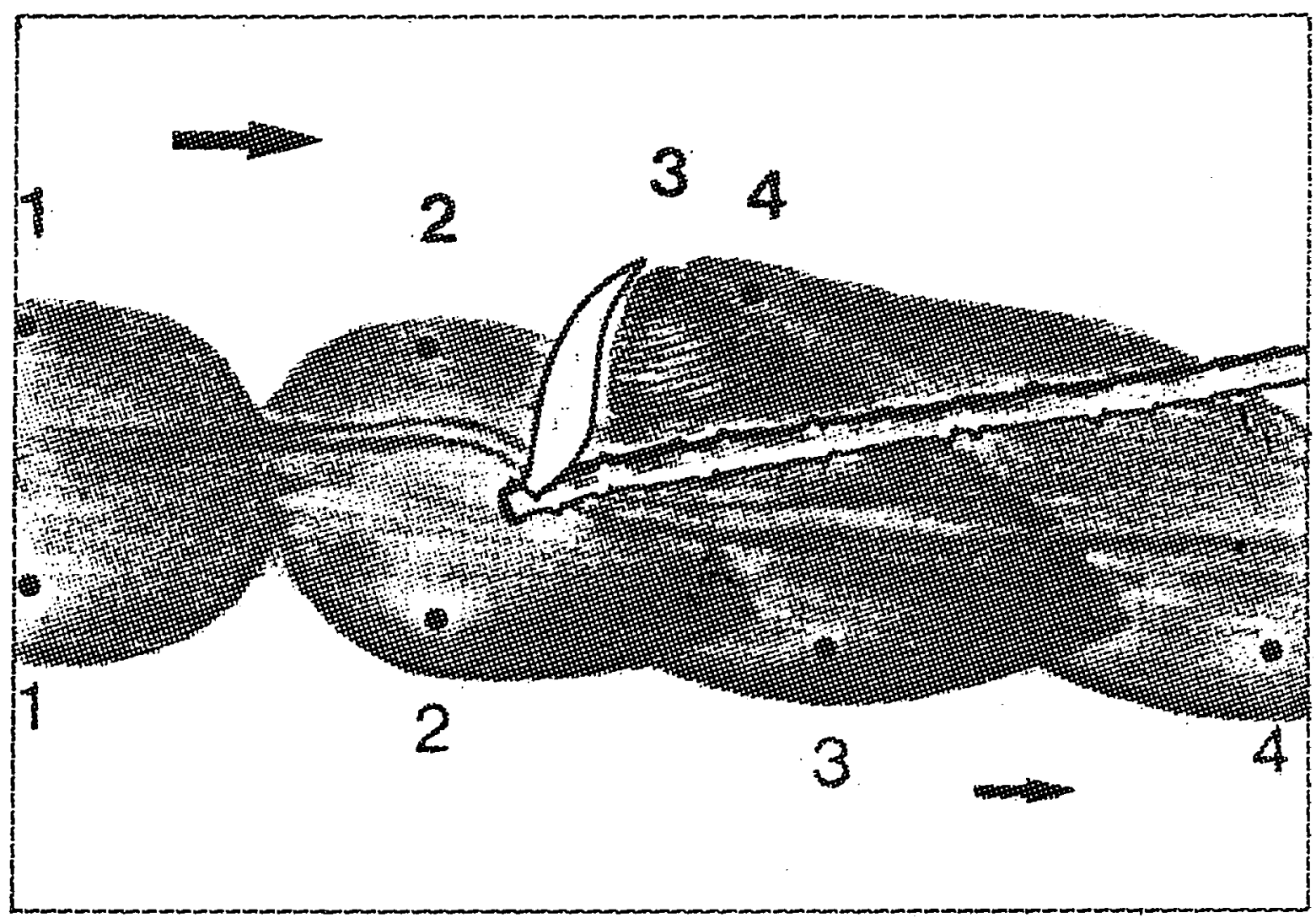

Fig. 7. Déplacements successifs (au 1/18 s.) d'une goutte de colorant frôlant l'échantillon : le freinage, côté tourbillons est évident.

Fig. 7. Successive shifting (at $1 / 18 \mathrm{~s}$.) of a drop of the dye skimming the sample : the braking system near the twirling is evident.

quelques nuances - qui n'ont d'intérêt que dañs un contexte d'études faunistiques - $F$. squamosa, en montage unifoliaire (Fig. 1) pris entre des formations tourbillonnaires assez similaires, engendre des rétrocourants comparables.

Ces nuances sont bien plus accentuées chez $F$. antipyretica. Plus que sa dimension supérieure, sa section en forme de $\mathrm{V}$, souvent très resserrée, distingue sa feuille des précédentes (Fig. 8. 3). Une morphologie si différente n'empêche pas la génération des rétrocourants. La convexité de la feuille face au courant apparaît donc ainsi comme la condition essentielle à la présence de ces formations tourbillonnaires.

Il faut pourtant noter, concernant $F$. antipyretica, une nette différence dans la distribution des vitesses à l'intérieur même de ces courants en retour sous-foliaires. En coloration ponctuelle ( $(2$.) les passages dans la partie évasée du limbe accusent des vitesses comparables à celles des deux précédentes espèces. Par contre, plus la partie axiale est resserrée, plus lent est le temps d'élimination totale du colorant-témoin en profondeur (après un temps suffisant en apport coloré). Une moyenne de $90 \mathrm{~s}$. a été repérée pour cette espèce, alors que celle-ci avoisinait $30 \mathrm{~s}$. pour $F$. squamosa et $15 \mathrm{~s}$. pour $P$. riparioides. Des vitesses sous-foliaires si différentes ne vont pas sans conséquences écologiques.

Les conséquences faunistiques de phyllotaxies différentes, non abordées ici, seraient intéressantes à rechercher.

\section{Conclusions}

En conclusion, une meilleure approche des courants sous-foliaires peut être envisagée. La présence de certains organismes, associée à des mesures de courants intercalaires rapides, est moins surprenante. C'est le cas pour Micrasema minimum, Protonemura sp, Aturus elongatus, inventoriés dans des échantillons de strate de mousse accusant des vitesses internes dépassant $100 \mathrm{ou} 150 \mathrm{~cm} / \mathrm{s}$ (Devantéry 1987). En fait, l'ha- 

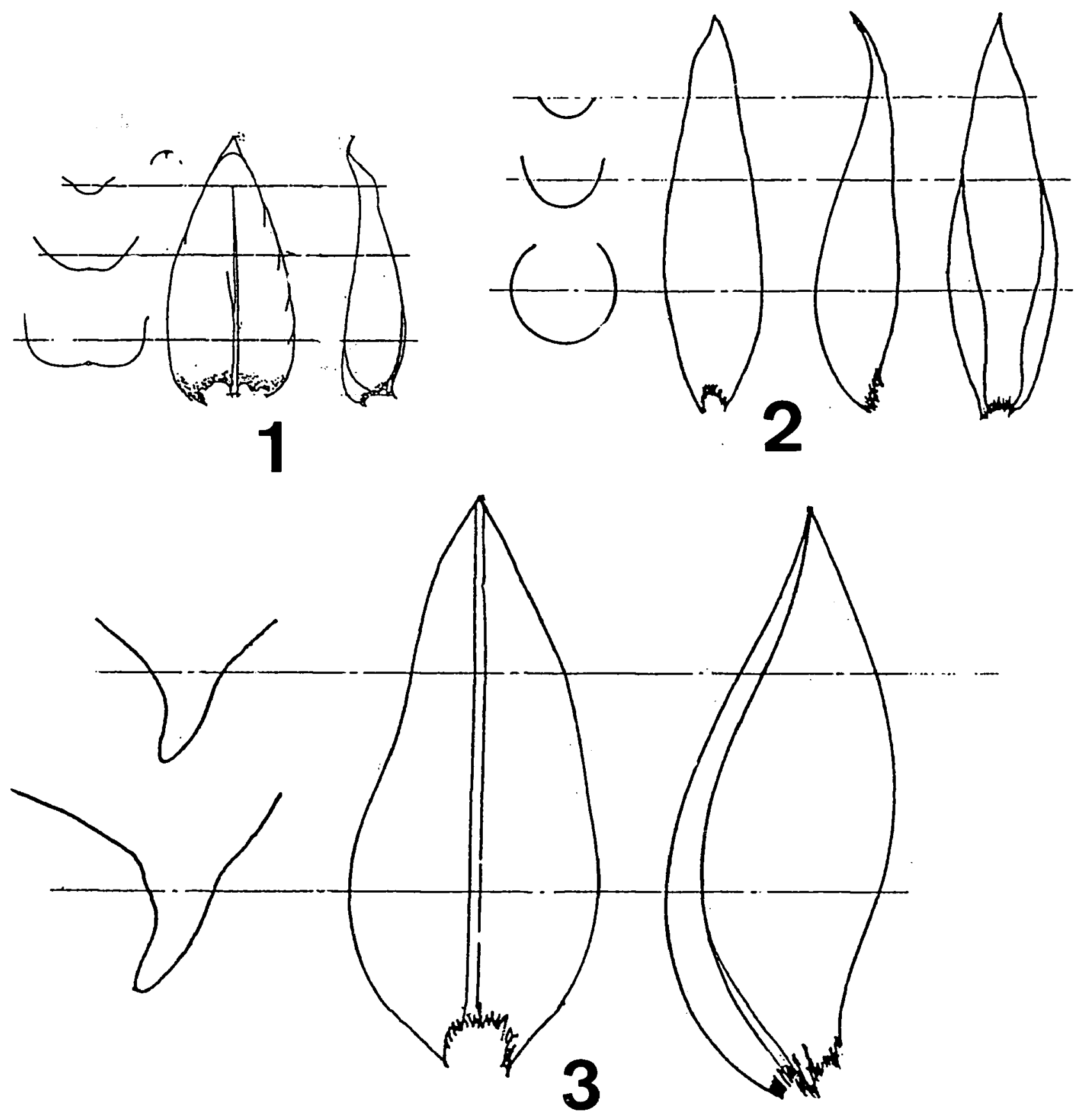

Fig. 8. Formes, dimensions et sections comparées d'échantillons de feuilles des 3 espèces observées :

1. Platyhypnidium riparioides (longueur $2,1 \mathrm{~mm}$ )

2. Fontinalis squamosa (longueur $3,6 \mathrm{~mm}$ )

3. Fontinalis antipyretica (longueur $5 ; 2 \mathrm{~mm}$ )

Fig. 8. Comparison of the form, dimensions and sections of samples of leaves of the 3 observed kinds :

1. Platyhypnidium riparioides $(2.1 \mathrm{~mm}$ long)

2. Fontinalis squamosa ( $3.6 \mathrm{~mm}$ long)

3. Fontinalis antipyretica $(5.2 \mathrm{~mm}$ long)

bitat effectif de ces organismes se situait sans doute dans des zones concernées par les freinages sous-foliaires.

Inversement, on comprend mieux que selon Scott (1958, in Décamps et al. 1972), des larves de Rhyacophila dorsalis «quand les vitesses s'élèvent à 40-50 $\mathrm{cm} / \mathrm{s}$ en pleine eau se réfugient sous les pierres et ne se trouvent sur les faces supérieures que lorsque celles-ci sont recouvertes de mousses». De plus, comme l'indiquent Pattée \& Bournaud (1970) à propos des planaires, «la théophilie est alors reliée à la sûreté des itinéraires choisis aux limites entre zones rapides et calmes». Nous constatons que cette limite est tout particulièrement 
tranchée dans de telles strates de mousses. Concernant les Hydracariens, animaux de petite taille, «le courant joue un rôle important dans la répartition des espèces pétricoles : celles-ci sont éliminées des mousses lorsque sa vitesse s'accroît; mais il ne paraît pas jouer un rôle important dans la répartition des espèces strictement muscicoles, au moins lorsqu'elles colonisent les Hypnacées» (Angelier et al. 1985).

C'est probablement là encore une question d'adaptation à ce choix d'itinéraires : il y a évitement de la partie convexe des feuilles et préférence pour les régions sous-fcliaires, bénéficiant des rétrocourants et de leurs influences modératrices. Les cavités foliaires à courant très ralenti chez $F$. antipyretica $(\S 4$.) nous ont apparu occasionnellement comme très appréciées par les Oligochètes.

Les observations in situ (Devantéry 1987) devaient nous apporter elles aussi quelques indices interrogateurs. Un plécoptère Protonemura aux premiers stades larvaires a été trouvé, fixé encore en fond de feuille, les mandibules positionnées précisément au point d'arrivée, côté insertion, du rétrocourant à rôle visiblement alimentaire. Notons que la forme de cette larve correspond, comme celle de maints taxons (Elmis, par exemple) dans cette position, à la morphologie foliaire de cette mousse, ainsi qu'à l'espace inter-tourbillonnaire axial. Une étude exhaustive dans ce sens ne manquerait pas d'intérêt. Plus couramment, des larves de Chironomidés ont été trouvées fixées en insertion dans le creux d'une feuille, construisant leur tube en direction de l'arrivée du rétrocourant pourvoyeur. Dans cette structure, les fonds de feuille envahis de pontes multiples n'apparaissent plus comme de simples refuges, mais bien comme des points nourriciers adaptés aux premiers stades de développement de la faune.

Le domaine des implications possibles tend à s'élargir si on considère que ces rétrocourants sélectionnent des éléments fins ou légers organiques - avec peut-être recyclages de déchets - ou minéraux, matériaux alimentaires de toute nature.

La vitesse du cours d'eau considéré peut même fournir des indications. Dès 1978, Johnson inventoriait en détritus végétaux «une proportion plus forte de particules plus petites dans les courants plus rapides» en un tel environnement de mousses. Nos observations en trois stations à différentes vitesses montraient, sur 16 mois, un colmatage en détritus végétaux nettement supérieur en vitesses lentes, alors qu'une certaine tendance accumulatrice en éléments sableux fins aux vitesses rapides avait aussi été décelée (Devantéry 1987).
Ces exemples nous ramènent ( $\$ 3.6$.) à ce décalage plus grand en courants rapides, entre vitesses intercalaires et rétrocourants, privilégiant ainsi les dépôts sous-foliaires proprement dits, très fins. L'effet inversé en courants lents est encore accru, concernant les débris végétaux, par le fait que l'encombrement moyen plus grand des particules joue encore un rôle accrocheur plus efficace dans la strate.

Du point de vue pratique, il est utile de souligner que ces ralentissements foliaires doivent nous rendre exigeants lors de l'étude d'échantillons de mousse : leur contenu intégral ne peut être récupéré que très difficilement au moyen de lavages en courants d'eau.

Le but du présent travail reste essentiellement d'établir une donnée mésologique utile. Il permet déjà d'entrevoir le facteur vital et stabilisant, dans les mousses aquatiques, représenté par ces freinages tourbillonnaires, pour les organismes mieux adaptés par leur forme, leur taille, leurs moeurs, à ces microcourants. Seule la multiplication des relations faune-milieu permettra de contrôler vraiment ces implications et leurs limites.

\section{Travaux cités}

Ambühl H. 1959. - Die Bedeutung der Strömung als ökologischer Factor. Schweiz. Z. Hydrol.,22 : 133-264.

Angelier E., Angelier M.L. \& Lauga J. 1985. - Recherches sur l'écologie des Hydracariens (Hydrachnellae, Acari) dans les eaux courantes. Annls Limnol. 21 : 25-64.

Bournaud M. 1963. - Le courant, facteur écologique et éthologique de la vie aquatique. Hydrobiologia, 1-2 : 125-165.

Décamps H., Capblancq J. \& Hirigoyen J.P. 1972. — Étude des conditions d'écoulement près du substrat en canal expérimental. Verh. Internat. Verein. Limnol. 18 : 718-725.

Devantéry P. 1987. - Action des courants sur la faune d'une mousse immergée : Platyhypnidium riparioides. Thèse dipl. Doct. Univ. Lyon I. 290 p.

Dorier A. \& Vaillant F. 1954. - Observations et expériences relatives à la résistance au courant de divers invertébrés aquatiques. Trav. lab. Hydrobiol. \& Pisc. Univ. de Grenoble 45-46 ann. 9-31.

Illies J., Rogler A. \& Meigering M.P.D. 1974. - Moderne Flusswasser - Experimenter Gerinne Ausbau der Flusstation Schlitz. Arch. Hydrobiol. 74-1 : 48-50.

Johnson T. 1978. - Aquatic mosses and stream metabolism in a North Swedish. river. Verh. Internat. Verein. Limnol. 20 : 1471 1477.

Larras J. 1974. - L'Hydraulique. Presses Universitaires de France, Paris : $128 \mathrm{p}$.

Pattée E. \& Bournaud M. 1970. - Etude expérimentale de la rhéophilie chez les Planaires triclades d'eau courante. Schweiz. Z. Hydrol. $32: 181-191$.

Scott D. 1958. - Ecological studies on the Trichoptera of the river Dean, Cheschire. Arch. Hydrobiol. 54 : 340-392.

Trivellato D. \& Décamps H. 1971. Dispositif expérimental pour l'étude du comportement d'espèces benthiques en eau courante. Annls Limnol. $7: 1$. 